\title{
Fatty acid signatures and stable isotopes as dietary indicators in North Sea seabirds
}

\author{
Anne Käkelä ${ }^{1}$, Robert W. Furness ${ }^{2}$, Andrew Kelly ${ }^{3,4}$, Ursula Strandberg ${ }^{1}$, \\ Susan Waldron ${ }^{3,5}$, Reijo Käkelä ${ }^{1, *}$ \\ ${ }^{1}$ Faculty of Biosciences, University of Joensuu, PO Box 111, 80101 Joensuu, Finland \\ ${ }^{2}$ Institute of Biomedical and Life Sciences, Graham Kerr Building, University of Glasgow, Glasgow, G12 8QQ, UK \\ ${ }^{3}$ Scottish Universities Environmental Research Centre, Scottish Enterprise Technology Park, Rankine Avenue, \\ East Kilbride, G75 0QF, UK \\ ${ }^{4}$ Present address: RSPCA, Stapeley Grange Wildlife Centre, London Road, Nantwich, Cheshire CW5 7JW, UK \\ ${ }^{5}$ Present address: Geographical and Earth Sciences, University of Glasgow, Glasgow G12 8QQ, UK
}

\begin{abstract}
Fatty acid signatures (FAS) of plasma and stable isotopes of carbon $\left(\delta^{13} \mathrm{C}\right)$ and nitrogen $\left(\delta^{15} \mathrm{~N}\right)$ of red blood cells were determined in northern gannets Morus bassanus, great skuas Stercorarius skua, shags Phalacrocorax aristotelis, and common guillemots Uria aalge from colonies in the North Sea (collected 2002 to 2003) in order to compare foraging ecologies, and especially to assess the extent to which birds feed on demersal or pelagic prey. The biochemical markers in great skua and gannet indicated that these species feed at a relatively high trophic level, and high variance, especially in great skua, demonstrated either a wide range of food types, individual dietary specialisation or both. The biochemical markers suggested that demersal fish are important constituents of great skua and gannet diets, and thus changes in fisheries discard rates probably influence these populations. In contrast, clear pelagic characteristics and low variance in the markers showed that the diet of common guillemots and shags is pelagic and varies little in composition. Comparison with the reference FAS data for North Sea fish confirmed the dependence of common guillemots on few shoaling pelagic species of fish, probably mainly young sandeels Ammodytes marinus.
\end{abstract}

KEY WORDS: Fatty acid signatures $\cdot$ Stable isotopes $\cdot$ Fisheries $\cdot$ Foraging ecology $\cdot$ Common murre Seabirds $\cdot$ North Sea

Resale or republication not permitted without written consent of the publisher

\section{INTRODUCTION}

Changes at a lower trophic level of marine food webs caused by human activities or by natural environmental change may result in marked declines of populations at a higher level. In general, specialist feeders are considered more vulnerable than opportunists that can switch prey. The Northeast Atlantic, including the North Sea, is presently facing a large restructuring of food webs, and both fisheries and changed weather conditions appear to play a part. Especially large changes have been recorded, and are expected to continue, in seabird communities of the North Sea (Mitchell et al. 2004, Votier et al. 2004, Wanless et al. 2005).

In the North Sea, discards from trawl fisheries (i.e. demersal species of fish) have become a key food for scavenging seabirds (Garthe et al. 1996). Without fisheries, demersal species such as haddock Melanogrammus aeglefinus and whiting Merlangius merlangus would not be available for these scavenging birds, as the fish live too deep. Scavenging seabird populations have increased several fold (Mitchell et al. 2004), possibly due to improved overwinter condition (Hüppop \& 
Wurm 2000) and breeding performance resulting from the availability of this extra food, but there are now large reductions in the amounts of demersal fish made available to scavenging seabirds (Votier et al. 2004).

In addition to the long-term and year-to-year changes in availability of discards, the seabird populations of North Sea are affected by availability of highenergy shoaling pelagic fish (Furness \& Tasker 2000, Wanless et al. 2005). Populations of the sandeel Ammodytes marinus in the northern North Sea collapsed in 2002. The reduced availability of sandeels and the lowenergy values of the remaining specimens caused unprecedented breeding failure of common guillemots Uria aalge and many other seabirds of the northern part of the North Sea in 2004 (Mavor et al. 2005, Wanless et al. 2005). Better understanding of seabird foraging ecology and seabird-fisheries interactions may aid conservation and ecosystem management.

Seabirds and other free-ranging marine animals are challenging objects for studies of foraging ecology. In many cases direct observations of their prey are not possible, and analyses of stomach contents, regurgitates, and especially pellets may overestimate the dietary items that leave clearly detectable and easily identified hard remains (Votier et al. 2003). These snapshots of the foods eaten may also fail to reveal the average diet of the species, and data collection tends to be possible only during the breeding season. In addition, the importance of different prey in terms of assimilated food and energy content is difficult to estimate based on hard parts. The digestion efficiencies of North Sea seabirds are also related to their feeding ecology and digestive tract morphology (Hilton et al. 2000). Thus complementary biochemical methods, including determination of stable isotope ratios of nitrogen $\left({ }^{15} \mathrm{~N} /{ }^{14} \mathrm{~N}, \delta^{15} \mathrm{~N}\right)$ and carbon $\left({ }^{13} \mathrm{C} /{ }^{12} \mathrm{C}, \delta^{13} \mathrm{C}\right)$ and studies of fatty acid signatures (FAS), are gaining increased application in marine foraging ecology (Bearhop et al. 2001, Dahl et al. 2003, Andersen et al. 2004).

$\delta^{15} \mathrm{~N}$ is usually used to study the trophic position of a consumer in a food web, since there is a pronounced and predictable stepwise increase in the value in each trophic transfer (Hobson \& Welch 1992). $\delta^{13} \mathrm{C}$ can reveal contributions of different sources to the diet of the consumer, e.g. terrestrial versus aquatic or benthic versus pelagic (Hobson et al. 1995, Sydeman et al. 1997). As with $\delta^{15} \mathrm{~N}$, there is generally an increase of $\delta^{13} \mathrm{C}$ from diet to consumer, but in contrast to the pattern with nitrogen, the enrichment in carbon isotopic signature is small (Hobson \& Welch 1992).

FAS may give more detailed information on the most likely prey items than the stable isotope ratios (Dahl et al. 2003, Andersen et al. 2004). Although the FAS provide information on the ingested prey, the metabolic processes of all organisms at lower trophic levels of the food web have contributed to the final FAS. This creates a need for laborious calibration studies to reveal the metabolic modifications of the FAS from diet to consumer tissues (Iverson et al. 2004, Käkelä et al. 2005). The FAS contains some 30 to 70 different fatty acids of varying relative amounts. Marine organisms have diverse fatty acid compositions, characterised by several long chain polyunsaturated fatty acids (PUFA) originating from phytoplankton, and several doublebond positional isomers of long chain monounsaturated fatty acids (MUFA) originating from zooplankton wax esters (Ackman et al. 1968, Saito \& Kotani 2000, Budge et al. 2002). FAS have been utilized largely to compare FAS of marine mammals and those of their potential prey. There are only a few studies comparing FAS of seabirds and their potential prey (Raclot et al. 1998, Dahl et al. 2003, Connan et al. 2005, Käkelä et al. 2006).

In this study we sampled blood from 4 species of seabirds at colonies in the northwestern North Sea (northern gannet Morus bassanus, common guillemot Uria aalge, shag Phalacrocorax aristotelis and great skua Stercorarius skua) using the plasma for FAS analysis and the red blood cells (RBC) for analysis of $\delta^{13} \mathrm{C}$ and $\delta^{15} \mathrm{~N}$. For dietary comparison, we used FAS and stable isotope data from the quantitatively most important demersal and pelagic fish species of the North Sea and from captive herring gulls Larus argentatus fed these fish (Käkelä et al. 2005, A. Kelly et al. unpubl.). The goals were to (1) use the biochemical markers to describe the feeding ecology of each species, (2) compare the information from the biochemical markers with previous knowledge on the feeding behaviour of the species obtained by conventional methods, and (3) compare the results from and the usefulness of the FAS and stable isotope approaches.

\section{MATERIALS AND METHODS}

Samples. Blood samples of about $0.5 \mathrm{ml}$ were taken (under British Home Office licence) by venipuncture from the median metatarsal vein. In spring 2002, samples were taken from 76 adult great skuas, 14 adult common guillemots, and 14 shag chicks, at Foula, Shetland, UK. In spring 2003, the material was supplemented with samples from 93 adult great skuas from 4 colonies in different parts of Shetland (Foula, Fair Isle, Hermaness and Fetlar), and from 11 gannet chicks (Bass Rock, east Scotland, UK). To prevent blood clotting, EDTA was added. The blood samples were centrifuged at $9000 \times g$ and $4^{\circ} \mathrm{C}$ for $15 \mathrm{~min}$. The separated plasma was removed for FAS analysis and the RBC pellets for stable isotope analysis. Both types of samples were stored at $-80^{\circ} \mathrm{C}$. 
Existing FAS and stable isotope reference data for whole fish homogenates of demersal (plaice Pleuronectes platessa, haddock and whiting) and pelagic (sardine Sardina pilchardus, mackerel Scomber scombrus and lean juveniles of herring Clupea harengus) fish species from the North Sea (Käkelä et al. 2005, A. Kelly et al. unpubl.) were supplemented by analyzing whole fish homogenates of 23 sandeels Ammodytes marinus captured by the Centre for Environment, Fisheries and Aquaculture Science, Lowestoft, in the central North Sea $\left(54^{\circ} 30^{\prime} \mathrm{N}, 0^{\circ} 30^{\prime} \mathrm{E}\right)$ in June 2005. The sandeels were divided into 2 groups according to the fishing device used, i.e. a group caught in a Granton trawl ( $\mathrm{N}=12,16$ to $18 \mathrm{~cm}, 11$ to $20 \mathrm{~g}$ ) and a group caught in a sandeel dredge $(\mathrm{N}=11,10$ to $18 \mathrm{~cm}, 3$ to $13 \mathrm{~g}$ ).

Fatty acid analysis. The plasma samples were thawed and an aliquot of $200 \mu \mathrm{l}$ from each was dried under nitrogen and immediately transmethylated by heating with $1 \% \mathrm{H}_{2} \mathrm{SO}_{4}$ in methanol under nitrogen atmosphere. The fatty acid methyl esters (FAME) formed were extracted with hexane in 2 steps. The dried and concentrated FAME of total lipids were identified and quantified by gas-liquid chromatography using both flame ionization (FID) and mass detection (6890N network GC with FID and 5973 MSD, Agilent) as detailed previously (Käkelä et al. 2005). The FAME samples of the sandeel total lipids were prepared using the same procedure, but with subsamples of whole fish homogenates instead of plasma.

Stable isotope analysis. RBC and samples of potential dietary fish were freeze dried and ground to powder using an agate mortar and pestle. Approximately $0.7 \mathrm{mg}$ of homogenised samples were sealed in a tin capsule $(4 \times 6 \mathrm{~mm})$ and analysed using continuous flow isotope ratio mass spectrometry (CF-IRMS) (Delta Plus $\mathrm{XP}$, Thermoelectron). Isotope ratios were expressed in parts per thousand (\%o), according to the following equation: $\delta X=\left[\left(R_{\text {sample }} / R_{\text {standard }}\right)-1\right] \times 1000$, where $X$ is ${ }^{15} \mathrm{~N}$ or ${ }^{13} \mathrm{C}$ and $R$ is the corresponding ratio of ${ }^{15} \mathrm{~N} /{ }^{14} \mathrm{~N}$ or ${ }^{13} \mathrm{C} /{ }^{12} \mathrm{C}$. $R_{\text {standard }}$ for ${ }^{15} \mathrm{~N} /{ }^{14} \mathrm{~N}$ is atmospheric nitrogen, $\mathrm{AIR}$, and $R_{\text {standard }}$ for ${ }^{13} \mathrm{C} /{ }^{12} \mathrm{C}$ is PDB (Pee Dee Belemnite). Typical precision and accuracy of measurement was $0.2 \%$ for $\delta^{15} \mathrm{~N}$ and $0.1 \%$ for $\delta^{13} \mathrm{C}$.

Although the addition of EDTA may potentially affect the carbon and nitrogen isotopic signatures of the blood, EDTA accounted for $<5 \%$ of total sample volume. As EDTA is water soluble, much of it would probably have been removed in the blood plasma. Nevertheless, we assessed the contribution of EDTA using blood collected from captive seabirds (Käkelä et al. 2005). Samples were split into 2 aliquots, with one washed in phosphate-buffered saline $19 \%$ saline $(0.155 \mathrm{M} \mathrm{NaCl})$ and 10 to $25 \mathrm{mM}$ phosphate, $\mathrm{pH}$ 7.4). Samples were then centrifuged at $9000 \times g$ for $10 \mathrm{~min}$ and the supernatant removed. The red blood cells were re-suspended by vortex mixing and the process was repeated. Samples were freeze-dried, homogenised and prepared for stable isotope analysis as described above. Subsequent stable isotope analysis was on unwashed blood samples, since effects of washing were found to be negligible (see 'Results'). The lipid component of the fish homogenates was not removed, which in the fatty pelagic fish has a lowering effect on $\delta^{13} \mathrm{C}$ values.

Statistical analysis. The relationships between the FAS in different plasma samples were studied by multivariate principal component analysis (PCA) using SIRIUS 6.5 software package (Pattern Recognition Systems). The data were standardized, and the relative positions of the samples or variables plotted using 2 new coordinates, the principal components PC 1 and PC 2, which represent the largest and second largest variance among the samples or variables. One-way analyses of variance (ANOVA) followed by the Newman-Keuls tests were used to study the differences in the seabird stable isotopes and calculated indicative fatty acid ratios. The statistical significance of differences in the variances of these markers was tested by the $F$-test.

\section{RESULTS}

Washing the blood had no significant effect on the mean $\delta^{13} \mathrm{C}(-19.8 \pm 1.9$ versus $-19.2 \pm 2.0$ for washed and unwashed samples, respectively) or the mean $\delta^{15} \mathrm{~N}$ $(11.0 \pm 3.0$ versus $11.9 \pm 2.9$, respectively $)$. The maximum offsets caused by EDTA were $0.57 \%$ for $\delta^{13} \mathrm{C}$ and $0.63 \%$ for $\delta^{15} \mathrm{~N}$ for the mean value of the blood samples (Table 1). The EDTA contribution to the isotopic signature of the blood was therefore negligible relative to the field signatures.

The stable isotope data showed species-specific differences, with gannets most ${ }^{15} \mathrm{~N}$ - and ${ }^{13} \mathrm{C}$-enriched,
Table 1 . The \%o offset in $\delta^{13} \mathrm{C}$ and $\delta^{15} \mathrm{~N}$ caused by addition of 4 different contributions of EDTA to the samples. $\delta^{13} \mathrm{C}$ and $\delta^{15} \mathrm{~N}$ for blood are mean values

\begin{tabular}{|c|c|c|c|c|c|c|c|}
\hline \multirow[b]{2}{*}{$\begin{array}{c}\text { EDTA } \\
(-30.2 \% \circ)\end{array}$} & \multicolumn{3}{|c|}{$\delta^{13} \mathrm{C}-$} & \multirow[b]{2}{*}{$\begin{array}{l}\text { EDTA } \\
(0.3 \%)\end{array}$} & \multirow{2}{*}{$\begin{array}{c}\delta^{1} \\
\text { Blood } \\
(12.9 \% \text { o })\end{array}$} & \multirow{2}{*}{$\begin{array}{l}\mathrm{N} \\
\text { Blood } \\
+ \text { EDTA }\end{array}$} & \multirow{2}{*}{$\%$ offset } \\
\hline & $\begin{array}{c}\text { Blood } \\
(-18.9 \%)\end{array}$ & $\begin{array}{c}\text { Blood } \\
+ \text { EDTA }\end{array}$ & $\%$ offset & & & & \\
\hline 0.001 & 0.999 & -18.91 & 0.01 & 0.001 & 0.999 & 12.89 & 0.01 \\
\hline 0.005 & 0.995 & -18.96 & 0.06 & 0.005 & 0.995 & 12.84 & 0.06 \\
\hline 0.01 & 0.99 & -19.01 & 0.11 & 0.01 & 0.99 & 12.77 & 0.13 \\
\hline 0.05 & 0.95 & -19.47 & 0.57 & 0.05 & 0.95 & 12.27 & 0.63 \\
\hline
\end{tabular}


followed by skuas, guillemots and shags (Fig. 1A,B). Variance in guillemot $\delta^{15} \mathrm{~N}$ was small compared to the variances in skuas and shags ( $\mathrm{p}<0.01, F$-test). Variances in the shag and guillemot $\delta^{13} \mathrm{C}$ were very small (Figs. 1B \& 2C). The gannet and especially skua showed significantly larger variance $(\mathrm{p}<0.001, F$-test). With the exception of haddock, demersal fish were isotopically enriched relative to pelagic fish (Table 2). The prey samples analysed were broadly similar to the samples collected in earlier studies of these
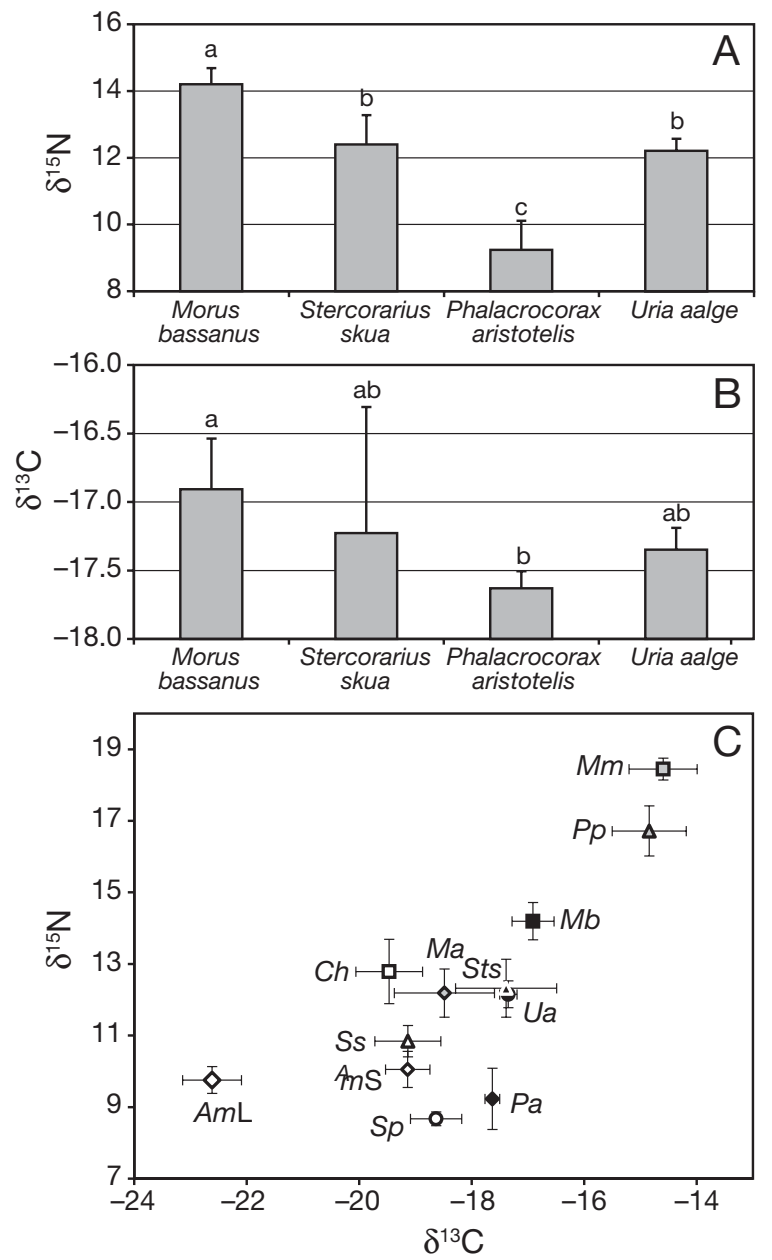

Fig. 1. Stable isotope ratios (means $+\mathrm{SD})$ of $(\mathrm{A})$ nitrogen $(\mathrm{p}<$ $0.0001,1$-way ANOVA) and (B) carbon ( $<<0.04,1$-way ANOVA) in the red blood cells of gannets Morus bassanus $(\mathrm{N}=11)$, great skuas Stercorarius skua $(\mathrm{N}=169)$, shags Phalacrocorax aristotelis $(\mathrm{N}=14)$ and common guillemots Uria aalge $(\mathrm{N}=14)$. Bars with different letters differ at $\mathrm{p}<0.05$ (Newman-Keuls test). (C) Stable isotope ratios (means \pm SD) of the red blood cells of birds and homogenates of potential dietary fish plotted together for comparison. Mb: Morus bassanus; Sts: S. skua; Pa: P. aristotelis; Ua: U. aalge, Ma: Melanogrammus aeglefinus; Mm: Merlangius merlangus; $\mathrm{Pp}$ : Pleuronectes platessa, Ch: Clupea harengus; Ss: Scomber scombrus; Sp: Sardina pilchardus; AmL: Ammodytes marinus large individuals, AmS: A. marinus small individuals
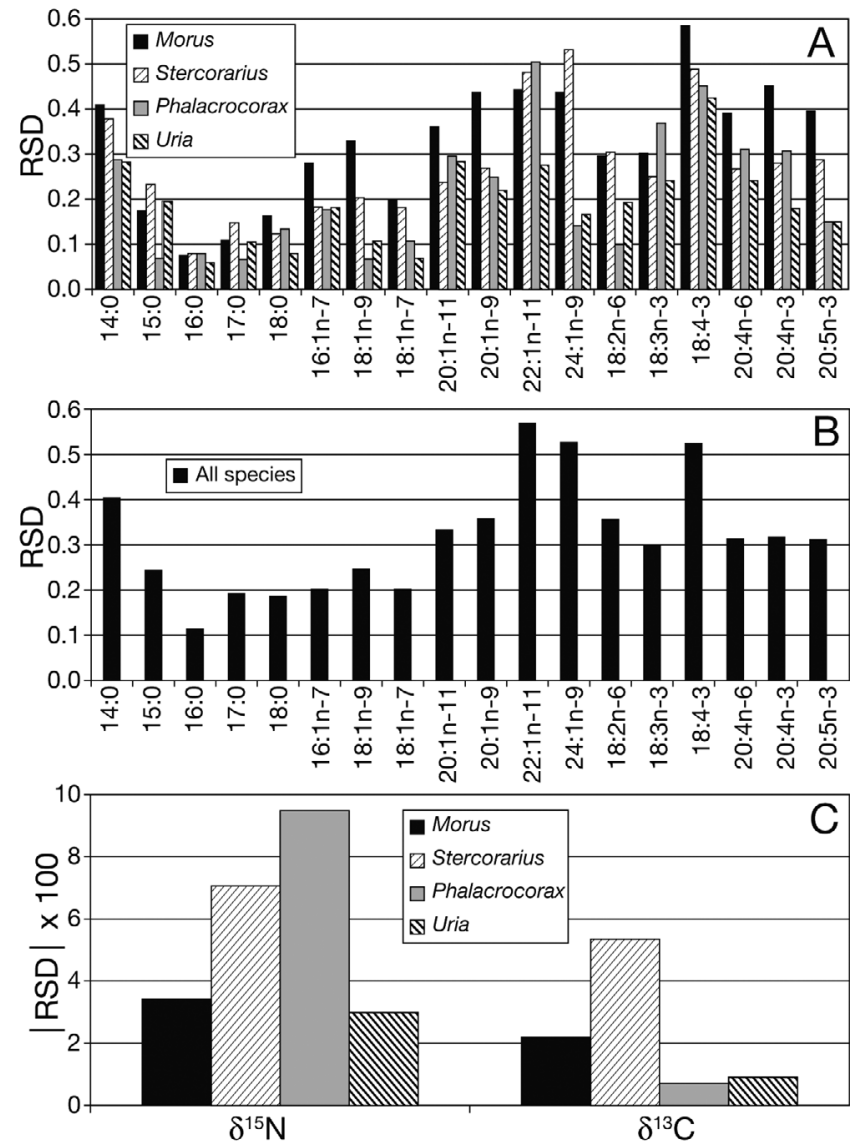

Fig. 2. (A) Relative variation (RSD = SD/mean, coefficient of variation) in the proportions ( $\mathrm{mol} \%$ ) of individual fatty acids in the plasma of gannets Morus bassanus $(\mathrm{N}=11)$, great skuas Stercorarius skua $(\mathrm{N}=169)$, shags Phalacrocorax aristotelis $(\mathrm{N}=14)$ and common guillemots Uria aalge $(\mathrm{N}=14)$, and (B) the respective variations in the combined data from all 4 species. Fatty acids that were present with at least $0.3 \mathrm{~mol} \%$ in 1 species and $0.15 \mathrm{~mol} \%$ in all species were included. (C) RSD of the stable isotope ratios of nitrogen and carbon in the plasma of the birds (expressed as absolute value $\times 100$ )

breeding colonies or others elsewhere in the North Atlantic, although whiting in this study is substantially more ${ }^{15} \mathrm{~N}$-enriched than in other studies where the signature is more similar to that of haddock (cf. Thompson et al. 1999, Bearhop et al. 2001). A comparison of stable isotope values of birds and pelagic and demersal fish (Fig. 1C) showed that gannet had the most demersal isotope signature, and shag the most pelagic signature. Great skua and guillemot had intermediate compositions.

The FAS in the seabird plasma comprised 48 individual fatty acids with a proportion of more than $0.1 \mathrm{~mol} \%$ in at least one species (Table 3 ). The relative amounts of each fatty acid varied considerably within and between the species (Fig. 2A,B). Among the quantita- 
Table 2. Major fatty acids, lipid content, and $\delta^{13} \mathrm{C}$ and $\delta^{15} \mathrm{~N}$ in homogenates of whole fish (median lengths in parentheses) from different demersal (haddock Melanogrammus aeglefinus, whiting Merlangius merlangus, flatfish spp., mainly plaice Pleuronectes platessa) and pelagic (herring Clupea harengus, sardine Sardina pilchardus, mackerel Scomber scombrus and sandeel Ammodytes marinus) species from the North Sea (means of 6 individuals). Large and small sandeels were analysed separately. The proportions are expressed as mol\%. Fatty acids that exceeded on average $0.5 \mathrm{~mol} \%$ in at least one species were listed.

Except for sandeel, the data are adapted from Käkelä et al. (2005). Sandeel data are from fish collected in June 2005

\begin{tabular}{|c|c|c|c|c|c|c|c|c|}
\hline & $\begin{array}{l}\text { Haddock } \\
(26 \mathrm{~cm})\end{array}$ & $\begin{array}{l}\text { Whiting } \\
(22 \mathrm{~cm})\end{array}$ & $\begin{array}{l}\text { Flatfish } \\
(18 \mathrm{~cm})\end{array}$ & $\begin{array}{l}\text { Herring } \\
(15 \mathrm{~cm})\end{array}$ & $\begin{array}{l}\text { Sardine } \\
(13 \mathrm{~cm})\end{array}$ & $\begin{array}{c}\text { Mackerel } \\
(30 \mathrm{~cm})\end{array}$ & $\begin{array}{l}\text { Sandeel } \\
(17 \mathrm{~cm})\end{array}$ & $\begin{array}{l}\text { Sandeel } \\
(13 \mathrm{~cm})\end{array}$ \\
\hline $14: 0$ & 3.2 & 1.2 & 2.9 & 5.4 & 8.9 & 7.4 & 9.1 & 3.9 \\
\hline $15: 0$ & 0.8 & 0.6 & 0.9 & 0.5 & 0.8 & 0.7 & 0.5 & 0.5 \\
\hline $16: 0$ & 15.2 & 17.9 & 15.8 & 20.5 & 19.4 & 15.9 & 16.4 & 22.4 \\
\hline $17: 0$ & 0.6 & 0.9 & 0.9 & 0.3 & 0.6 & 0.5 & 0.2 & 0.5 \\
\hline $17: 0$ iso & 0.5 & 0.7 & 0.9 & 0.2 & 0.1 & 0.3 & 0.1 & 0.2 \\
\hline 17:0 anteiso & 0.3 & 0.6 & 0.8 & 0.1 & 0.1 & 0.1 & 0.1 & 0.1 \\
\hline $18: 0$ & 4.0 & 6.3 & 5.1 & 2.5 & 3.0 & 3.0 & 2.2 & 5.2 \\
\hline $16: 1 n-9$ & 0.5 & 0.4 & 0.9 & 0.3 & 0.2 & 0.2 & 0.1 & 0.3 \\
\hline $16: 1 n-7$ & 4.9 & 3.0 & 6.2 & 3.5 & 10.2 & 5.3 & 7.2 & 3.9 \\
\hline $17: 1 \mathrm{n}-8$ & 0.7 & 0.7 & 0.8 & 0.2 & $\begin{array}{r}10.2 \\
0.2\end{array}$ & 0.4 & 0.1 & 0.3 \\
\hline $18: 1 n-13$ & 0.2 & 0.9 & 1.4 & Trace & Trace & Trace & 0.1 & Trace \\
\hline $18: 1 n-9+11$ & 12.9 & 8.8 & 6.5 & 9.7 & 5.7 & 11.4 & 4.8 & 7.1 \\
\hline $18: 1 n-7$ & 3.7 & 4.8 & 5.2 & 2.8 & 2.4 & 2.3 & 1.5 & 2.3 \\
\hline $18: 1 n-5$ & 0.5 & 0.6 & 0.3 & 0.5 & 0.2 & 0.4 & 0.3 & 0.5 \\
\hline $20: 1 n-11$ & 1.3 & 0.8 & 1.6 & 0.7 & 0.7 & 0.7 & 0.6 & 0.3 \\
\hline $20: 1 n-9$ & 2.6 & 1.1 & 0.9 & 5.2 & 5.1 & 8.0 & 11.6 & 2.9 \\
\hline $20: 1 n-7$ & 1.0 & 1.0 & 1.7 & 0.2 & 0.2 & 0.3 & 0.2 & 0.1 \\
\hline $22: 1 n-11$ & 2.0 & 0.1 & 0.1 & 8.2 & 7.1 & 10.3 & 13.4 & 3.7 \\
\hline $22: 1 n-9$ & 0.3 & 0.2 & 0.1 & 0.4 & 0.5 & 0.9 & 1.3 & 0.3 \\
\hline $24: 1 n-9$ & 0.4 & 0.7 & 0.1 & 0.6 & 0.5 & 0.8 & 0.7 & 1.0 \\
\hline $16: 2 n-4$ & 0.2 & 0.2 & 0.4 & 0.2 & 0.9 & 0.3 & 0.8 & 0.4 \\
\hline $16: 3 n-4$ & 0.1 & 0.1 & 0.3 & 0.2 & 0.4 & 0.2 & 0.6 & 0.2 \\
\hline $16: 4 n-1$ & 0.1 & 0.1 & 0.4 & 0.2 & 0.9 & 0.4 & 1.9 & 0.4 \\
\hline $18: 2 n-6$ & 1.6 & 0.5 & 0.6 & 1.7 & 1.3 & 1.6 & 1.5 & 1.5 \\
\hline $18: 3 n-3$ & 1.2 & 0.2 & 0.2 & 0.9 & 0.9 & 1.3 & 0.9 & 1.0 \\
\hline $18: 4 n-3$ & 2.4 & 0.3 & 0.5 & 1.4 & 1.7 & $\begin{array}{l}1.5 \\
3.8\end{array}$ & 3.4 & $\begin{array}{l}1.0 \\
1.6\end{array}$ \\
\hline $20: 2 n-6$ & 0.6 & 0.4 & 0.4 & 0.2 & 0.2 & 0.3 & 0.2 & 0.2 \\
\hline $20: 4 n-6$ & 1.7 & 3.3 & 3.3 & 1.0 & 0.7 & 0.7 & 0.4 & 0.9 \\
\hline $20: 4 n-3$ & 0.7 & 0.5 & 0.5 & 0.6 & 0.7 & 1.1 & 0.5 & 0.5 \\
\hline $20: 5 n-3$ & 12.0 & 13.8 & 19.3 & 8.2 & 9.5 & 5.9 & 8.2 & 11.3 \\
\hline $21: 5 n-3$ & 0.3 & 0.3 & 0.6 & 0.2 & 0.3 & 0.3 & 0.4 & 0.4 \\
\hline $22: 4 n-6$ & 0.6 & 2.1 & 2.0 & 0.3 & 0.2 & 0.2 & 0.4 & 0.2 \\
\hline $22: 5 n-6$ & 0.4 & 0.5 & 0.5 & 0.3 & 0.3 & 0.3 & 0.1 & 0.2 \\
\hline $22: 5 n-3$ & 1.2 & 3.6 & 5.4 & 0.9 & 1.1 & 1.3 & 0.6 & 0.7 \\
\hline $22: 6 n-3$ & 16.7 & 17.4 & 6.7 & 18.7 & $\begin{array}{l}1.1 \\
11.4\end{array}$ & 9.1 & 6.2 & 20.8 \\
\hline Lipid \% fresh wt & $2.0 \pm 0.7$ & $1.0 \pm 0.4$ & $1.0 \pm 0.4$ & $\begin{array}{l}10.7 \\
1.8 \pm 0.6\end{array}$ & $10.8 \pm 3.0$ & $8.3 \pm 1.2$ & $6.0 \pm 1.8$ & $1.5 \pm 0.4$ \\
\hline$\delta^{13} \mathrm{C}$ & $-18.5 \pm 0.9$ & $-14.6 \pm 0.6$ & $-14.8 \pm 0.7$ & $\begin{array}{r}1.0 \pm 0.0 \\
-19.5 \pm 0.6\end{array}$ & $\begin{array}{r}10.0 \pm 0.0 \\
-18.6 \pm 0.5\end{array}$ & $\begin{array}{r}0.0 \pm 1.2 \\
-19.1 \pm 0.6\end{array}$ & $\begin{array}{r}0.0 \pm 1.0 \\
-22.6 \pm 0.5\end{array}$ & $-19.1 \pm 0.4$ \\
\hline$\delta^{15} \mathrm{~N}$ & $\begin{array}{r}-10.5 \pm 0.9 \\
12.2 \pm 0.7\end{array}$ & $\begin{array}{r}-14.0 \pm 0.0 \\
18.4 \pm 0.3\end{array}$ & $\begin{array}{r}-14.0 \pm 0.7 \\
16.7 \pm 0.7\end{array}$ & $\begin{array}{r}19.0 \pm 0.0 \\
12.8 \pm 0.9\end{array}$ & $\begin{array}{r}-10.0 \pm 0.0 \\
8.7 \pm 0.2\end{array}$ & $\begin{array}{r}-19.1 \pm 0.0 \\
10.8 \pm 0.4\end{array}$ & $\begin{array}{r}-22.0 \pm 0.5 \\
9.8 \pm 0.4\end{array}$ & $\begin{array}{r}-19.1 \pm 0.4 \\
10.1 \pm 0.5\end{array}$ \\
\hline
\end{tabular}

tively most important fatty acids, the largest deviations (RSD $=\mathrm{SD} /$ mean, coefficient of variation) were found in long chain MUFA and PUFA. Potentially endogenous (see 'Discussion') saturated fatty acids (SFA) and MUFA had stable levels. The fatty acid with the least variation was 16:0. When the species were compared, the deviations in fatty acid percentages in general were much smaller in common guillemot and shag than in gannet and great skua (Fig. 2).

Using the 36 qualitatively most important fatty acids as loadings in the PCA, the plasma samples of gannets, great skuas and shags overlapped due to the large variation in each species (Fig. 3). The FAS of great skua samples scattered the most and spanned the whole range of the PC axes, but a theoretical average individual would have been located between gannets and shags. Some of the gannets were very rich in 20:4n-6, 18:1n-9 and 18:0. In the reference data for North Sea fish (Table 2), these fatty acids are characteristic of demersal species. In contrast, shags were relatively rich in 22:1n-11, 20:1n-9 and 14:0, which were abundant in most of the pelagic fish. The guillemot samples were situated tightly packed in the lower left corner of the plot (Fig. 3). On PC1 (explaining $41 \%$ of the variation) the range in the FAS of the other species was at least twice that of the common guillemots. The common 
Table 3. Fatty acid composition (mol\%) in the plasma of gannets Morus bassanus, great skuas Stercorarius skua, shags Phalacrocorax aristotelis and common guillemots Uria aalge from northwestern North Sea colonies. The fatty acids that exceeded on average $0.1 \mathrm{~mol} \%$ in at least one species are listed. 4,8,12-TMTD: 4,8,12-trimethyltridecanoic acid; phytanate: 3,7,11,15tetramethylhexadecanoic acid; NMID: nonmethyle-interrupted-dienoic acid

\begin{tabular}{|c|c|c|c|c|c|c|c|c|c|c|c|c|c|c|c|c|}
\hline & \multicolumn{4}{|c|}{$\begin{array}{c}\text { Morus bassanus } \\
(\mathrm{N}=11)\end{array}$} & \multicolumn{4}{|c|}{$\begin{array}{l}\text { Stercorarius skua } \\
\quad(\mathrm{N}=169)\end{array}$} & \multicolumn{4}{|c|}{$\begin{array}{l}\text { Phalacrocorax aristotelis } \\
\qquad(\mathrm{N}=14)\end{array}$} & \multicolumn{4}{|c|}{$\begin{array}{l}\text { Uria aalge } \\
(\mathrm{N}=14)\end{array}$} \\
\hline & Mean & $\mathrm{SD}$ & Min & Max. & Mean & $\mathrm{SD}$ & Min. & Max. & Mean & $\mathrm{SD}$ & Min. & Max. & Mean & $\mathrm{SD}$ & Min. & Max. \\
\hline $14: 0$ & 1.68 & 0.69 & 0.69 & 2.73 & 2.33 & 0.88 & 0.66 & 5.71 & 2.74 & 0.79 & 1.51 & 4.17 & 1.14 & 0.32 & 0.76 & 2.01 \\
\hline $14: 0$ iso & 0.04 & 0.04 & 0.01 & 0.12 & 0.03 & 0.03 & 0.00 & 0.17 & 0.10 & 0.07 & 0.04 & 0.24 & 0.02 & 0.02 & 0.01 & 0.08 \\
\hline $15: 0$ & 0.31 & 0.05 & 0.21 & 0.38 & 0.36 & 0.08 & 0.17 & 0.63 & 0.47 & 0.03 & 0.40 & 0.52 & 0.26 & 0.05 & 0.17 & 0.35 \\
\hline $15: 0$ iso & 0.08 & 0.04 & 0.04 & 0.18 & 0.15 & 0.09 & 0.01 & 0.67 & 0.49 & 0.33 & 0.16 & 1.30 & 0.12 & 0.02 & 0.09 & 0.18 \\
\hline $16: 0$ & 32.95 & 2.47 & 27.45 & 36.16 & 24.86 & 1.94 & 17.98 & 29.32 & 30.30 & 2.37 & 27.38 & 34.14 & 25.62 & 1.52 & 23.62 & 28.60 \\
\hline $17: 0$ & 0.32 & 0.04 & 0.26 & 0.37 & 0.44 & 0.06 & 0.31 & 0.60 & 0.55 & 0.04 & 0.50 & 0.61 & 0.28 & 0.03 & 0.22 & 0.33 \\
\hline $17: 0$ iso & 0.09 & 0.02 & 0.07 & 0.14 & 0.14 & 0.03 & 0.06 & 0.29 & 0.16 & 0.03 & 0.12 & 0.21 & 0.16 & 0.03 & 0.12 & 0.22 \\
\hline 17:0 anteiso & 0.07 & 0.02 & 0.04 & 0.09 & 0.08 & 0.01 & 0.01 & 0.13 & 0.09 & 0.01 & 0.07 & 0.12 & 0.08 & 0.01 & 0.06 & 0.10 \\
\hline $18: 0$ & 23.46 & 3.81 & 15.76 & 28.04 & 14.49 & 1.80 & 9.56 & 18.46 & 14.28 & 1.92 & 10.45 & 16.82 & 14.29 & 1.12 & 12.97 & 16.36 \\
\hline $18: 0$ iso & 0.13 & 0.03 & 0.08 & 0.18 & 0.15 & 0.03 & 0.08 & 0.23 & 0.19 & 0.03 & 0.13 & 0.26 & 0.10 & 0.01 & 0.08 & 0.13 \\
\hline 19:0 & 0.09 & 0.02 & 0.05 & 0.13 & 0.10 & 0.02 & 0.04 & 0.17 & 0.10 & 0.01 & 0.08 & 0.12 & 0.05 & 0.01 & 0.04 & 0.07 \\
\hline $20: 0$ & 0.17 & 0.04 & 0.10 & 0.23 & 0.20 & 0.05 & 0.08 & 0.43 & 0.15 & 0.05 & 0.04 & 0.22 & 0.17 & 0.02 & 0.13 & 0.20 \\
\hline 4,8,12-TMTD & 0.05 & 0.07 & 0.01 & 0.23 & 0.11 & 0.12 & 0.00 & 0.81 & 0.16 & 0.07 & 0.05 & 0.31 & 0.23 & 0.10 & 0.07 & 0.46 \\
\hline Phytanate & 0.08 & 0.06 & 0.02 & 0.21 & 0.22 & 0.17 & 0.03 & 1.06 & 0.23 & 0.08 & 0.14 & 0.44 & 0.44 & 0.12 & 0.25 & 0.70 \\
\hline $15: 1 \mathrm{n}-8$ & 0.12 & 0.10 & 0.05 & 0.39 & 0.03 & 0.02 & 0.01 & 0.15 & 0.05 & 0.02 & 0.03 & 0.10 & 0.03 & 0.01 & 0.02 & 0.06 \\
\hline 16:1n-11 & 0.09 & 0.04 & 0.03 & 0.19 & 0.21 & 0.04 & 0.11 & 0.36 & 0.15 & 0.02 & 0.12 & 0.19 & 0.08 & 0.02 & 0.04 & 0.10 \\
\hline $16: 1 n-9$ & 0.16 & 0.03 & 0.11 & 0.20 & 0.19 & 0.16 & 0.09 & 1.51 & 0.25 & 0.03 & 0.19 & 0.32 & 0.25 & 0.11 & 0.14 & 0.57 \\
\hline $16: 1 n-7$ & 2.21 & 0.62 & 1.29 & 3.30 & 2.48 & 0.45 & 1.37 & 3.74 & 2.88 & 0.51 & 2.07 & 3.81 & 1.89 & 0.34 & 1.36 & 2.60 \\
\hline $16: 1 n-5$ & 0.10 & 0.04 & 0.05 & 0.19 & 0.13 & 0.03 & 0.06 & 0.23 & 0.15 & 0.02 & 0.12 & 0.19 & 0.09 & 0.01 & 0.07 & 0.11 \\
\hline $17: 1 \mathrm{n}-8$ & 0.15 & 0.06 & 0.07 & 0.32 & 0.22 & 0.04 & 0.03 & 0.40 & 0.15 & 0.01 & 0.13 & 0.17 & 0.19 & 0.02 & 0.16 & 0.22 \\
\hline 17:1 branched & 0.02 & 0.01 & 0.01 & 0.03 & 0.05 & 0.02 & 0.02 & 0.10 & 0.10 & 0.02 & 0.07 & 0.14 & 0.06 & 0.02 & 0.03 & 0.08 \\
\hline 18:1n-9+11 & 12.96 & 4.25 & 8.38 & 22.65 & 15.67 & 3.17 & 11.65 & 32.15 & 9.11 & 0.62 & 8.23 & 10.03 & 20.66 & 2.21 & 15.93 & 23.42 \\
\hline $18: 1 n-7$ & 1.32 & 0.26 & 0.87 & 1.81 & 1.68 & 0.31 & 0.20 & 3.39 & 2.10 & 0.23 & 1.87 & 2.71 & 1.26 & 0.09 & 1.09 & 1.42 \\
\hline $18: 1 n-5$ & 0.15 & 0.03 & 0.10 & 0.19 & 0.20 & 0.04 & 0.02 & 0.29 & 0.19 & 0.03 & 0.15 & 0.23 & 0.18 & 0.02 & 0.14 & 0.21 \\
\hline 20:1n-11 & 0.63 & 0.23 & 0.31 & 0.96 & 1.04 & 0.25 & 0.44 & 1.71 & 0.68 & 0.20 & 0.44 & 1.02 & 0.32 & 0.09 & 0.23 & 0.54 \\
\hline $20: 1 n-9$ & 1.37 & 0.60 & 0.61 & 2.50 & 2.18 & 0.58 & 0.87 & 4.06 & 2.72 & 0.68 & 1.75 & 3.87 & 0.56 & 0.12 & 0.38 & 0.76 \\
\hline $20: 1 \mathrm{n}-7$ & 0.06 & 0.03 & 0.03 & 0.15 & 0.13 & 0.03 & 0.03 & 0.23 & 0.13 & 0.02 & 0.10 & 0.17 & 0.08 & 0.01 & 0.06 & 0.08 \\
\hline $22: 1 n-11$ & 0.58 & 0.26 & 0.28 & 1.22 & 1.71 & 0.82 & 0.39 & 4.92 & 1.50 & 0.75 & 0.59 & 2.86 & 0.29 & 0.08 & 0.17 & 0.42 \\
\hline $22: 1 n-9$ & 0.08 & 0.06 & 0.01 & 0.18 & 0.15 & 0.11 & 0.02 & 0.73 & 0.14 & 0.08 & 0.06 & 0.37 & 0.05 & 0.01 & 0.04 & 0.07 \\
\hline $24: 1 n-9$ & 0.24 & 0.10 & 0.13 & 0.43 & 0.57 & 0.30 & 0.05 & 1.89 & 0.69 & 0.10 & 0.54 & 0.89 & 0.41 & 0.07 & 0.23 & 0.51 \\
\hline $16: 2 n-4$ & 0.11 & 0.03 & 0.05 & 0.16 & 0.14 & 0.05 & 0.00 & 0.32 & 0.19 & 0.09 & 0.00 & 0.33 & 0.05 & 0.02 & 0.02 & 0.10 \\
\hline $16: 3 n-4$ & 0.04 & 0.02 & 0.01 & 0.07 & 0.07 & 0.04 & 0.00 & 0.27 & 0.15 & 0.06 & 0.06 & 0.23 & 0.05 & 0.02 & 0.03 & 0.10 \\
\hline $16: 4 n-1$ & 0.06 & 0.04 & 0.00 & 0.15 & 0.15 & 0.15 & 0.01 & 1.75 & 0.29 & 0.13 & 0.11 & 0.54 & 0.07 & 0.03 & 0.03 & 0.13 \\
\hline $18: 2 n-6$ & 1.06 & 0.31 & 0.66 & 1.67 & 1.87 & 0.57 & 0.85 & 4.38 & 1.31 & 0.13 & 1.06 & 1.50 & 0.84 & 0.16 & 0.67 & 1.24 \\
\hline $18: 2 n-4$ & 0.05 & 0.01 & 0.03 & 0.08 & 0.09 & 0.03 & 0.04 & 0.22 & 0.12 & 0.02 & 0.05 & 0.14 & 0.07 & 0.01 & 0.05 & 0.09 \\
\hline $18: 3 n-3$ & 0.16 & 0.05 & 0.08 & 0.25 & 0.37 & 0.09 & 0.15 & 0.67 & 0.43 & 0.16 & 0.20 & 0.67 & 0.35 & 0.09 & 0.23 & 0.49 \\
\hline $18: 4 n-3$ & 0.19 & 0.11 & 0.04 & 0.43 & 0.54 & 0.26 & 0.07 & 1.59 & 0.82 & 0.37 & 0.28 & 1.34 & 0.58 & 0.24 & 0.27 & 1.04 \\
\hline $20: 2 n-6$ & 0.11 & 0.03 & 0.06 & 0.15 & 0.13 & 0.04 & 0.05 & 0.38 & 0.20 & 0.03 & 0.16 & 0.28 & 0.13 & 0.02 & 0.11 & 0.17 \\
\hline 20:2NMID & 0.11 & 0.05 & 0.03 & 0.20 & 0.09 & 0.05 & 0.01 & 0.23 & 0.08 & 0.01 & 0.06 & 0.11 & 0.02 & 0.01 & 0.01 & 0.05 \\
\hline $20: 3 n-6$ & 0.08 & 0.02 & 0.05 & 0.13 & 0.17 & 0.05 & 0.00 & 0.35 & 0.14 & 0.03 & 0.09 & 0.20 & 0.09 & 0.02 & 0.06 & 0.12 \\
\hline $20: 4 n-6$ & 6.43 & 2.52 & 3.23 & 10.39 & 6.03 & 1.61 & 2.86 & 12.34 & 3.61 & 1.12 & 2.03 & 5.84 & 3.68 & 0.89 & 2.19 & 5.17 \\
\hline $20: 4 n-3$ & 0.22 & 0.10 & 0.12 & 0.38 & 0.43 & 0.12 & 0.08 & 0.87 & 0.32 & 0.10 & 0.16 & 0.51 & 0.58 & 0.10 & 0.45 & 0.84 \\
\hline $20: 5 n-3$ & 5.63 & 2.22 & 2.63 & 10.54 & 10.33 & 2.94 & 3.23 & 17.72 & 8.12 & 1.22 & 5.74 & 10.14 & 13.90 & 2.09 & 11.36 & 17.59 \\
\hline $21: 5 n-3$ & 0.01 & 0.03 & 0.00 & 0.08 & 0.11 & 0.05 & 0.02 & 0.28 & 0.14 & 0.06 & 0.03 & 0.21 & 0.13 & 0.04 & 0.08 & 0.21 \\
\hline $22: 4 n-6$ & 0.09 & 0.03 & 0.06 & 0.14 & 0.08 & 0.04 & 0.02 & 0.20 & 0.11 & 0.04 & 0.06 & 0.18 & 0.10 & 0.04 & 0.07 & 0.22 \\
\hline $22: 5 n-6$ & 0.08 & 0.04 & 0.00 & 0.12 & 0.33 & 0.16 & 0.11 & 1.05 & 0.40 & 0.08 & 0.27 & 0.59 & 0.36 & 0.11 & 0.17 & 0.55 \\
\hline $22: 5 n-3$ & 0.60 & 0.22 & 0.32 & 0.94 & 0.80 & 0.18 & 0.25 & 1.49 & 0.76 & 0.08 & 0.63 & 0.90 & 0.57 & 0.09 & 0.39 & 0.78 \\
\hline $22: 6 n-3$ & 4.21 & 1.13 & 2.63 & 5.92 & 6.84 & 0.97 & 3.55 & 9.86 & 10.45 & 0.57 & 9.18 & 11.62 & 7.92 & 0.87 & 6.52 & 9.71 \\
\hline
\end{tabular}

guillemot samples were clearly separated from the other species, and enriched in 20:5n-3, phytanate, 22:6n-3, 20:4n-3 and 18:4n-3 compared to the other species.

The plasma FAS data were also plotted together with our previous calibration FAS from captive herring gulls fed various pelagic and demersal fish (Käkelä et al. 2005) (Fig. 4). Some gannet and great skua individuals had clearly demersal FAS. The shags had mainly pelagic FAS, and so did most of the great skuas. The guillemot samples were on the pelagic side, but had 


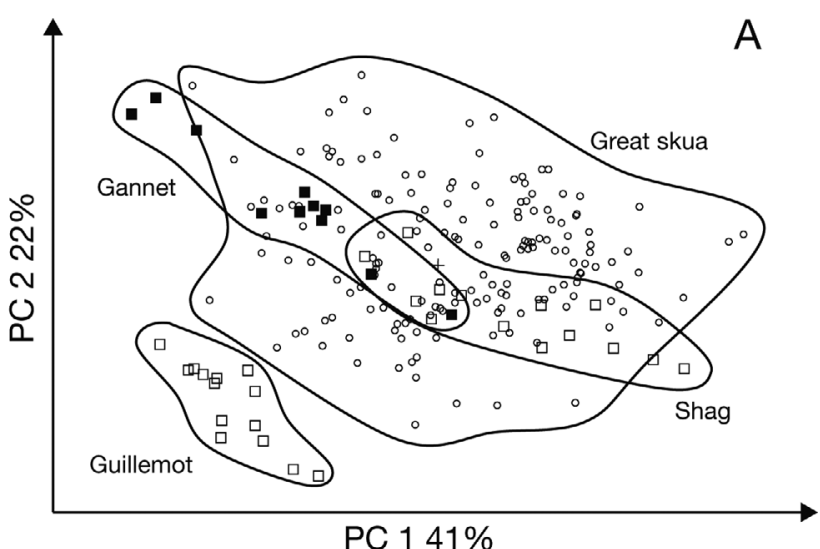

PC $141 \%$

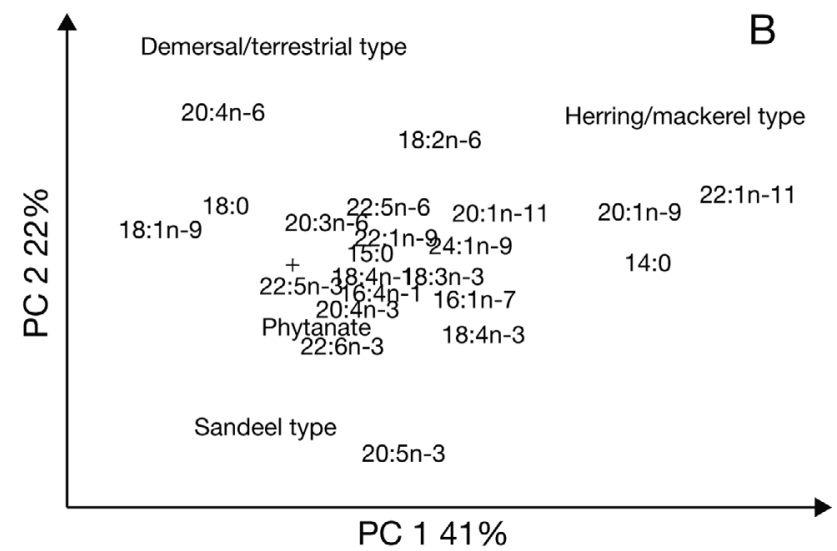

Fig. 3. (A) Principal component analysis of the plasma samples of gannets Morus bassanus $(\mathrm{N}=11)$, great skuas Stercorarius skua $(\mathrm{N}=169)$, shags Phalacrocorax aristotelis $(\mathrm{N}=14)$ and common guillemots Uria aalge $(\mathrm{N}=14)$. (B) The 36 quantitatively most import fatty acids were used as loadings. The fatty acids characteristic for demersal/terrestrial diets grouped into upper left corner of the plot; those characteristic for herring/ mackerel type of fish on the right, and those characteristic of small sandeels at the bottom near the PC 1 axis. Several fatty acids with small eigenvalues close to origo (+) are not shown

smaller amounts of 22:1n-11, 20:1n-9 and 14:0. Guillemot samples separated clearly from the pelagic specimens of shag and great skua that had large proportions of these fatty acids.

The specific PUFA ratio (20:4n-6 to the sum of $18: 3 n-3$, 18:4n-3 and 20:5n-3) used in an earlier herring gull calibration study (Käkelä et al. 2005) to differentiate demersal versus pelagic diet was applied to these data from wild seabirds and their potential prey (Fig. 5A,C). The contribution of the potentially demersal or terrestrial PUFA decreased and that of pelagic PUFA increased in the sequence gannet-great skua-shag-guillemot. The variances decreased in the same order (all significantly different from each other, $\mathrm{p}<0.01$ or $\mathrm{p}<0.001$, $F$-test). The proportion of 20:1n-11, 20:1n-9 and 22:1n-11 in the MUFA totals (an indication of pelagic copepods in the diet; see 'Discussion' and Fig. 5D) increased sequen-
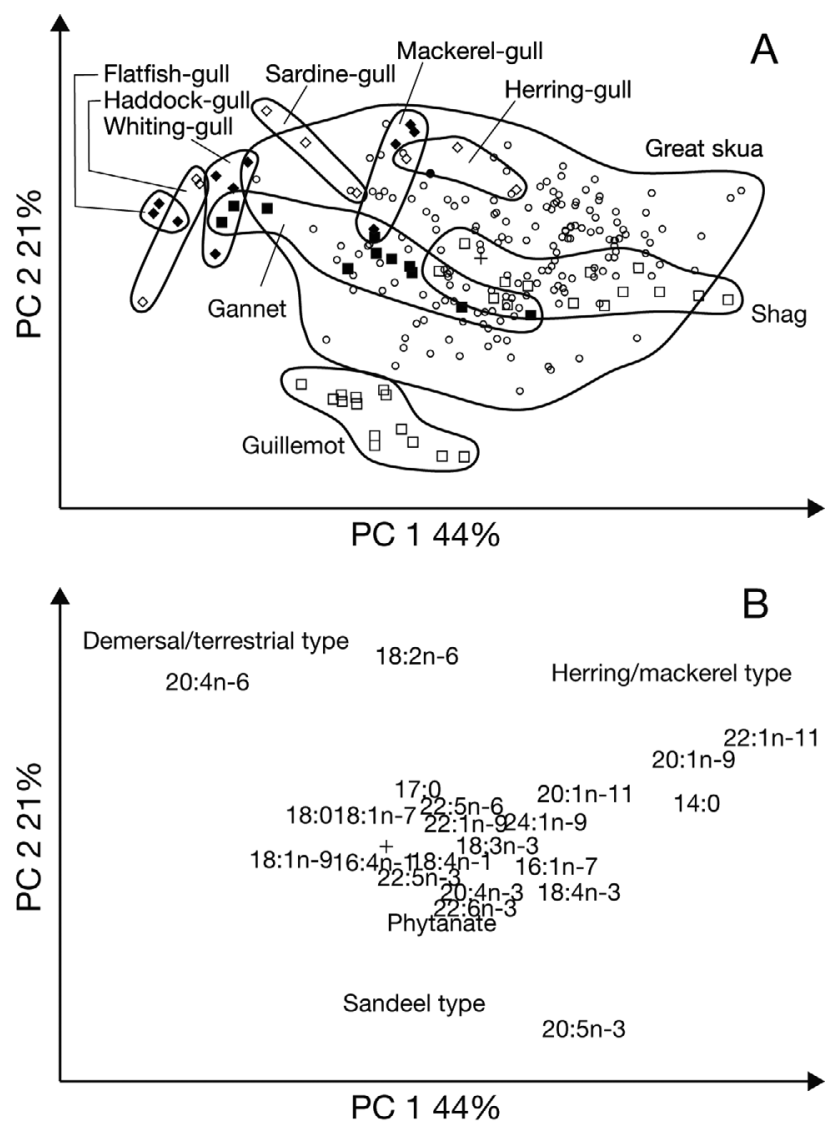

Fig. 4. Principal component analysis of the plasma samples of gannets Morus bassanus $(\mathrm{N}=11)$, great skuas Stercorarius skua $(\mathrm{N}=169)$, shags Phalacrocorax aristotelis $(\mathrm{N}=14)$ and common guillemots Uria aalge $(\mathrm{N}=14)$ corresponding to sample data shown in Fig. 2 (with additional data from captive herring gulls fed different demersal and pelagic fish, e.g. flatfish-gull, haddock-gull; Käkelä et al. 2005). Several fatty acids with small eigenvalues close to origo (+) are not shown. (A) Relationships of the samples; (B) relationships of the fatty acids, i.e. variables

tially from gannet to great skua to shag (Fig. 5B). However, the plasma samples of common guillemots had very little of these MUFA. In addition, the variance of this marker was significantly smaller in the guillemot than in the other species ( $p<0.001, F$-test).

\section{DISCUSSION}

\section{Variability of the diet}

FAS of vertebrate tissues comprise both diet-derived and endogenously synthesized fatty acids. Thus, to interpret the dietary information of the FAS correctly, the metabolic origin of the fatty acids should be considered. Most of the quantitatively important MUFA and SFA can be synthesized endogenously. The main prod- 

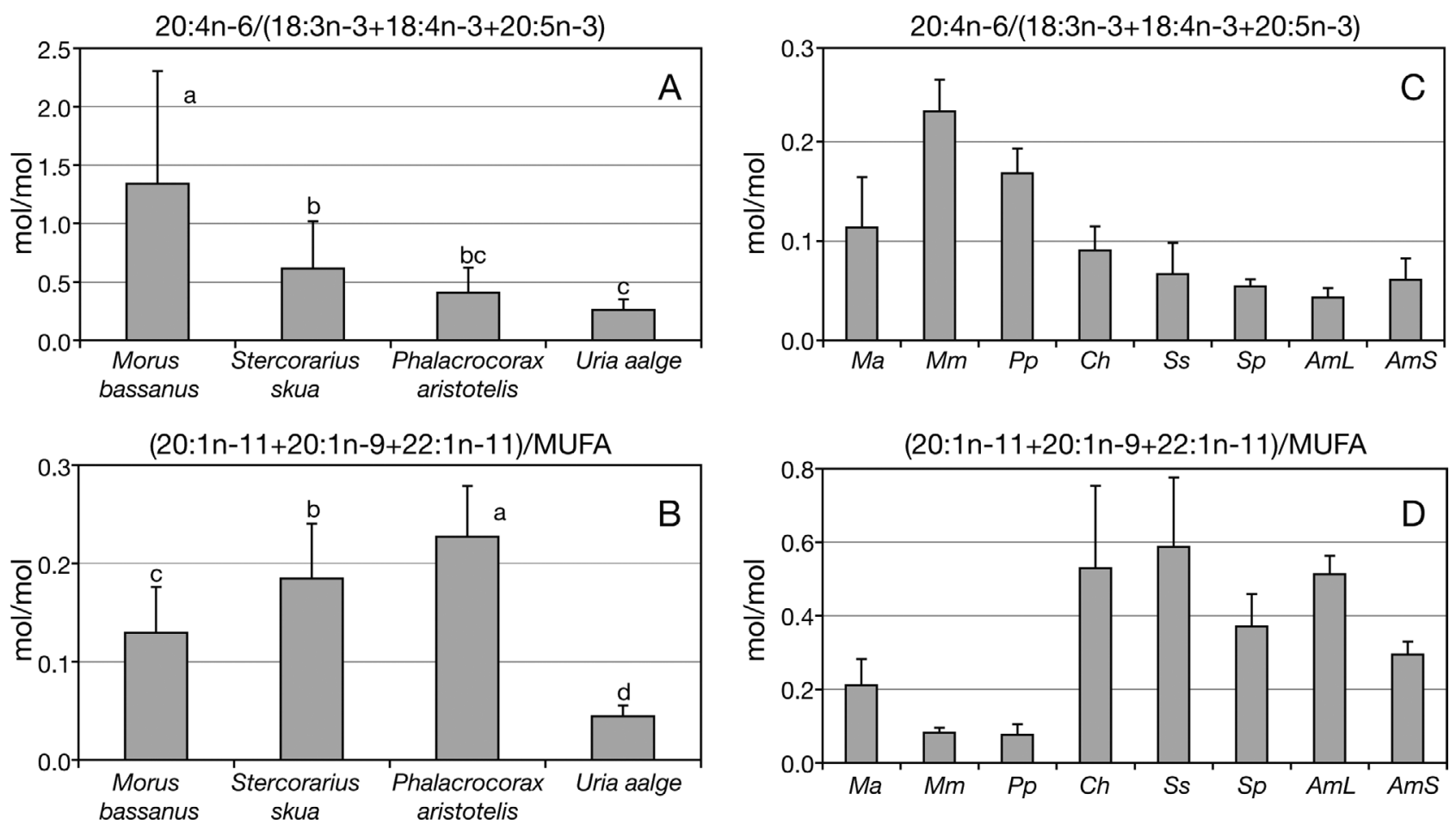

Fig. 5. Specific fatty acid ratios in the plasma of gannets Morus bassanus $(\mathrm{N}=11)$, great skuas Stercorarius skua ( $\mathrm{N}=169)$, shags Phalacrocorax aristotelis $(\mathrm{N}=14)$ and common guillemots Uria aalge $(\mathrm{N}=14)$ (means $+\mathrm{SD})$ : $(\mathrm{A})$ polyunsaturated fatty acids (PUFA) ratio 20:4n-6/(18:3n-3+18:4n-3+20:5n-3), high values of which indicate large demersal/terrestrial influence in the diet $(\mathrm{p}<0.0001$, 1-way ANOVA), and (B) ratio of the most important potentially copepod-derived monounsaturated fatty acids (MUFA) (20:1n-11+20:1n-9+22:1n-11) to MUFA total, high values of which indicate marked copepod-type zooplankton influence in the diet $(\mathrm{p}<0.0001,1$-way ANOVA). For both fatty acid ratios, the bars indicated with different letters differ at $\mathrm{p}<0.05$ (Newman-Keuls test). Corresponding ratios for total lipids in whole homogenates of potential demersal and pelagic fish prey are shown in (C) and (D) (abbreviations as in Fig. 1)

uct of cytosomal fatty acid synthethase is $16: 0$ and it can be desaturated to $16: 1 \mathrm{n}-7$ or elongated to $18: 0$, and then desaturated to $18: 1 \mathrm{n}-9$. Desaturation before elongation gives double bond positional isomer 18:1n-7. In addition, fatty acid synthethase can utilize alternative primers, e.g. propionate instead of acetate and, consequently, $17: 0$ is produced instead of 16:0. Thus, theoretically the levels of these and other minor endogenous fatty acids in tissue lipids can be adjusted by means of biosynthesis. However, if the dietary supply is sufficient, de novo synthesis of these fatty acids is not required. In contrast, most PUFA in vertebrate tissues are of dietary origin, derived from diet as such or as their precursors (Ackman \& Cunnane 1992). In addition, long chain MUFA of marine vertebrates originate from zooplankton, mainly copepod wax esters (Saito \& Kotani 2000).

The RSD of fatty acids in the seabird plasma, which can be used as an indication of diet variability of a species, fit well with the different origins of different fatty acids summarised above. The percentage of 16:0 had the smallest RSD in the FAS of the seabirds stud- ied, and was similar in each species (Fig. 2). The SFA shorter than 16:0, e.g. 14:0 and 15:0, had larger RSD, indicating stronger dietary influence. In MUFA, the long chain 20:1n-9 and 22:1n-11 originating from zooplankton wax esters had much higher RSD than the shorter MUFA, the levels of which can be adjusted by biosynthesis. The RSD of plasma PUFA were in general large. Among major PUFA, the levels of 22:6n-3 had the lowest RSD, probably because $22: 6 \mathrm{n}-3$ is needed for phospholipid membrane function in vertebrates tissues; thus the levels of this fatty acid are preserved and controlled (Ackman \& Cunnane 1992). Plentiful supply of n-3 PUFA from diet was revealed by elevated levels of $18: 4 n-3,20: 5 n-3$ and 18:3n-3, rather than as changes in the levels of 22:6n-3. The most important n-6 PUFA, 20:4n-6, characteristic of demersal fish and terrestrial prey also showed a large RSD. Thus, in the present data variability in the diet of the species studied was detected most efficiently in the plasma PUFA composition and long chain MUFA content.

The RSD pattern in the array of all quantitatively important fatty acids resembled closely the pattern 
found previously in captive herring gulls fed different pelagic or demersal fish (Käkelä et al. 2005). In the experiment with gulls, diet was the only reasonable source of deviation in plasma FAS, which lends confidence to the proposition that variation in plasma FAS in the North Sea seabirds studied was also mainly of dietary origin. Thus, a small RSD in an individual fatty acid in one species indicates either biosynthesis and/or stable supply of this fatty acid from diet, and large RSD of a fatty acid means dietary origin. On the other hand, large RSD of several fatty acids indicates varied diet and considerable foraging flexibility in a population. Accordingly, fatty acids of great skua showed a wide variation of diet among individual birds, while the parents of gannet chicks had specialized feeding behaviour. In the case of great skuas, this conclusion is consistent with previous research showing that these birds display both a wide spectrum of diets across a population, as well as large individual specialisation in diet (Phillips et al. 1997, Votier et al. 2003). Great skuas also have greater digestive efficiencies and gut retention times than shags and guillemots, suggesting that skuas have opportunistic feeding behaviour (Hilton et al. 2000). The diet of gannets has not been studied in detail, but the results of this biomarker study also suggest that this species either consumes a highly varied diet containing both pelagic and demersal fish, or different individuals/pairs specialize on different prey across this spectrum, or both. Common guillemots and shags had much smaller RSD of individual fatty acids than great skuas or gannets. Therefore, common guillemot diet must be rather homogenous and similar among individuals. This is concordant with the reported strong dependence of common guillemots and shags on small pelagic shoaling fish (Wanless et al. 2005). In a comparative study of North Atlantic seabird digestion, guillemots had the smallest average gut retention times among the 8 species studied (Hilton et al. 2000), which suggests that guillemots prey mostly on easily digested and high energy dense fish.

Carbon isotopic variances were large for gannet and great skua, but very small for shag and guillemot. In blood, lipid and carbohydrate typically comprise $<5 \%$ of the dry mass (Bearhop et al. 2002); thus, this variance more likely reflects dietary variance than intraspecies variation in blood lipid content. Increased carbon isotopic variance can arise from a wide range of prey consumed, but in constant amounts (Bearhop et al. 2004). However, the simplest interpretation is that dietary sources of carbon for the gannets and skuas were much more variable than those of guillemots and shags, and this is consistent with the interpretation deduced from FAS data.

\section{Quality of the diet}

The ${ }^{15} \mathrm{~N}$ - and ${ }^{13} \mathrm{C}$-enriched signatures of gannet and great skua reveal a consumption of dietary items more ${ }^{15} \mathrm{~N}$-enriched than that eaten by shag chicks. Thus the blood isotopic signature of the gannet and skuas is as expected if these species consume a greater amount of demersal prey items, which are generally ${ }^{15} \mathrm{~N}$ - and ${ }^{13} \mathrm{C}$-enriched (our Table 2, Sydeman et al. 1997). The shag chicks' blood isotopic composition suggests they consume isotopically depleted prey. An assumed trophic offset of $3 \%$ implies that shag chicks' diet comprises an isotopically depleted source additional to sandeels. The additional pelagic food of the shags may have been small Norway Pout Trisopterus esmarki or sprat Sprattus sprattus, seen occasionally in the area. There is a similarity in guillemot and gannet blood isotopic signatures. The trophic offset (an average of $3 \%$ is assumed) between guillemot and sandeels is such that guillemot isotopic signatures may reflect a diet dominated by sandeels, thus supporting the FAS data (discussed below) which show dependency on small individuals with low long chain MUFA content.

The plasma FAS provided more detailed information on bird diets than the stable isotope ratios. The high ratios of an important demersal PUFA to important pelagic PUFA (20:4n-6/[18:3n-3+18:4n-3+20:5n-3]) suggest that discarded demersal fish is of considerable importance to gannets and great skuas. The diet of great skua was shown by several conventional methods to consist of varied items including discarded demersal fish, pelagic fish, and occasional invertebrate and terrestrial prey (Votier et al. 2003). Similarly, this ratio suggests that shags and guillemots do not consume discarded demersal fish or terrestrial prey rich in 20:4n-6 or its precursors. As long chain MUFA (20:1n-11, 20:1n-9 and 22:1n-11 are the most abundant) originate mainly from copepod wax esters; their large percentages in bird plasma indicate feeding on zooplankton-consuming pelagic fish like herring and mackerel, or large sandeels (Table 3). Thus, birds with a high value of the specific PUFA ratio, indicating strong demersal influence in the diet, should also have small contents of the long chain MUFA. Using the same reasoning for Spitsbergen seabirds, common eider Somateria mollissima was linked to the benthic food chain by its high levels of 20:4n-6, while blacklegged kittiwake Rissa tridactyla and northern fulmar Fulmarus glacialis were linked to the pelagic food chain, primarily because of their high levels of 20:1n-9 and 22:1n-11 (Dahl et al. 2003). However, in this respect North Sea common guillemots were exceptional.

In PCA, common guillemots were separated clearly from the other species and had, in general, very small RSD for all fatty acids. Common guillemot FAS were 
rich in PUFA that are abundant in pelagic fish, but poor in pelagic long chain MUFA. This indicates a small contribution of wax ester copepods in their food chain, suggesting that herring, mackerel and other similar species feeding on copepods were not important constituents of guillemot diet. It should also be noted that individual fish at different ages and life stages have different diets, which results in large withinspecies variations of FAS. This was reported recently for herring and northern sandeel Ammodytes dubius from Alaskan and Nova Scotian waters (Budge et al. 2002, Iverson et al. 2002). In our FAS data from North Sea fish, the young and lean sandeels were rich in n-3 PUFA and contained little long chain MUFA, and these fish were thus potential prey of guillemots. In contrast, the large and fatty specimens of sandeel contain large amounts of long chain MUFA, and therefore cannot be an important prey for the guillemots. At least in the years of this study in Shetland the common guillemots appear, on the basis of the FAS data, to have been feeding almost entirely on small sandeels.

\section{Methodological remarks}

We showed that stable isotopes and FAS are useful in studying the foraging of wild seabirds. That the information provided by the stable isotopes and FAS was generally consistent and in line with results from conventional methods, encourages utilization of such markers in studying complex communities of seabirds. In the optimal case, seabird feeding habits are studied by combining conventional food analysis and the chemical markers. The 2 chemical markers refer in part to different metabolic routings. The FAS reflect solely lipid metabolism, while the stable isotopes, especially ${ }^{15} \mathrm{~N}$, refer to protein metabolism. When blood samples are used, the advantage of the stable isotopes of RBC over plasma FAS is that the stable isotopes represent a diet averaged over several weeks (Carleton \& Martínez del Rio 2005, A. Kelly et al. unpubl.) while the FAS may change totally a few days after a switch of diet (Käkelä et al. 2005). It has been reported that all major lipid classes of vertebrate plasma and even lipids extracted from whole blood reflect dietary fatty acid input, but with different halftimes of incorporation (Katan et al. 1997, Baylin et al. 2005). However, the information obtained from the stable isotopes is restricted to general trophic position of bird species and estimation on the relative importance of demersal and pelagic species in the diet. In contrast, FAS can provide specific information on the prey species and even on the age classes of fish consumed where, as in the case of sandeels, the FAS apparently changes considerably with fish size/age. Therefore, the best results are obtained when a considerable effort is made to sample a representative library of prey FAS, and when large numbers of birds are sampled to get reliable estimates of both the average food and the magnitude of individual variation. The question as to whether all bird individuals change prey regularly, or, alternatively, different individuals specialize on different types of prey would be best answered by sampling the same birds several times during the season.

Both stable isotope and FAS approaches need calibration studies to produce quantitative estimates on the composition of the prey. For example, lean and fatty fish diets likely affect bird FAS to different extents (Iverson et al. 2004, Käkelä et al. 2006). In addition to experiments where the prey is switched (Iverson et al. 2004, Käkelä et al. 2005), additional studies are needed in which different mixing ratios are used to test how additive the changes of FAS are. We have already carried out feeding experiments to test this with captive herring gulls. Determination of changes in seabird diets over time using FAS may also reveal changes in prey availability, species abundance and even age and size distributions of the prey populations. An additional method that has not yet been applied to seabirds is stable isotope analysis of individual fatty acids by gas chromatography-isotope ratio mass spectrometry. This approach has great potential to confirm the origin of each fatty acid component of the FAS.

Acknowledgements. This study was supported by EU contract Q5RS-2001-00839 'DISCBIRD'. We thank S. Votier, J. Crane, S. Bearhop and P. Arcos for trapping skuas for blood sampling, and J. Metcalfe, CEFAS, Lowestoft, for obtaining samples of North Sea sandeels for fatty acid and stable isotope analyses. R.W.F. was supported by a Leverhulme Fellowship.

\section{LITERATURE CITED}

Ackman RG, Cunnane C (1992) Long-chain polyunsaturated fatty acids. Sources, biochemistry and nutritional/clinical applications. Adv Appl Lipid Res 1:161-215

Ackman RG, Tocher CS, McLachlan J (1968) Marine phytoplankter fatty acids. J Fish Res Board Can 25:1603-1620

Andersen SM, Lydersen C, Grahl-Nielsen O, Kovacs KM (2004) Autumn diet of harbour seals (Phoca vitulina) at Prins Karls Forland, Svalbard, assessed via scat and fattyacid analyses. Can J Zool 82:1230-1245

Baylin A, Kim MK, Donovan-Palmer A, Siles X, Dougherty L, Tocco P, Campos H (2005) Fasting whole blood as a biomarker of essential fatty acid intake in epidemiologic studies: comparison with adipose tissue and plasma. Am J Epidemiol 162:373-381

Bearhop S, Thompson DR, Phillips RA, Waldron S and 5 others (2001) Annual variation in great skua diets: the importance of commercial fisheries and predation on seabirds revealed by combining dietary analysis. Condor 103:802-809

Bearhop S, Waldron S, Votier SC, Furness RW (2002) Factors that influence assimilation rates and fractionation of nitrogen and carbon stable isotopes in avian blood and feathers. Physiol Biochem Zool 75:451-458 
Bearhop S, Adams CE, Waldron S, Fuller RA, Macleod H (2004) Determining trophic niche width - a novel approach using stable isotope analysis. J Anim Ecol 73:1007-1012

Budge SM, Iverson SJ, Bowen WD, Ackman RG (2002) Among - and within - species variability in fatty acid signatures of marine fish and invertebrates on the Scotian Shelf, Georges Bank, and southern Gulf of St. Lawrence. Can J Fish Aquat Sci 59:886-898

Carleton SA, Martínez del Rio C (2005) The effect of coldinduced increased metabolic rate on the rate of ${ }^{13} \mathrm{C}$ and ${ }^{15} \mathrm{~N}$ incorporation in house sparrows (Passer domesticus). Oecologia 144:226-232

Connan M, Mayzaud P, Boutoute M, Weimerskirch H, Cherel Y (2005) Lipid composition of stomach oil in a procellariiform seabird Pufinus tenuirostis: implications for food web studies. Mar Ecol Prog Ser 290:277-290

Dahl TM, Falk-Petersen S, Gabrielsen GW, Sargent JR, Hop H, Millar RM (2003) Lipids and stable isotopes in common eider, black-legged kittiwake and northern fulmar: a trophic study from an Arctic fjord. Mar Ecol Prog Ser 256: 257-269

Furness RW, Tasker ML (2000) Seabird-fishery interactions: quantifying the sensitivity of seabirds to reductions in sandeel abundance, and identification of key areas for sensitive seabirds in the North Sea. Mar Ecol Prog Ser 202: 253-264

Garthe S, Camphuysen CJ, Furness RW (1996) Amounts of discards by commercial fisheries and their significance as food for seabirds in the North Sea. Mar Ecol Prog Ser 136:1-11

Hilton GM, Furness RW, Houston DC (2000) A comparative study of digestion in North Atlantic seabirds. J Avian Biol 31: $36-46$

Hobson FA, Welch HE (1992) Determination of trophic relationships within a high Arctic marine food web using $\delta^{13} \mathrm{C}$ and $\delta^{15} \mathrm{~N}$ analysis. Mar Ecol Prog Ser 84:9-18

Hobson KA, Ambrose WG Jr, Renaud PE (1995) Sources of primary production, benthic-pelagic coupling, and trophic relationships within the Northeast Water Polonya: insights fom $\delta^{13} \mathrm{C}$ and $\delta^{15} \mathrm{~N}$ analysis. Mar Ecol Prog Ser 128:1-10

Hüppop O, Wurm S (2000) Effects of winter fishery activities on resting numbers, food and body condition of large gulls Larus argentatus and L. marinus in the south-eastern North Sea. Mar Ecol Prog Ser 194:241-247

Iverson SJ, Frost KJ, Lang SLC (2002) Fat content and fatty acid composition of forage fish and invertebrates in Prince William Sound, Alaska: factors contributing to among and within species variability. Mar Ecol Prog Ser 241:161-181

Iverson SJ, Field C, Bowen WD, Blanchard W (2004) Quantitative fatty acid signature analysis: a new method of esti-

Editorial responsibility: Otto Kinne (Editor-in-Chief), Oldendorf/Luhe, Germany mating predator diets. Ecol Monogr 74:211-235

Käkelä R, Käkelä A, Kahle S, Becker PH, Kelly A, Furness RW (2005) Fatty acid signatures in plasma of captive herring gulls as indicators of demersal or pelagic fish diet. Mar Ecol Prog Ser 293:191-200

Käkelä A, Crane J, Votier SC, Furness RW, Käkelä R (2006) Fatty acid signatures as indicators of diet in great skuas Stercorarius skua, Shetland. Mar Ecol Prog Ser 319: 297-310

Katan MB, Deslypere JP, van Birgelen APJM, Penders M, Zegwaard M (1997) Kinetics of the incorporation of dietary fatty acids into serum cholesteryl esters, erythrocyte membranes, and adipose tissue: an 18-month controlled study. J Lipid Res 38:2012-2022

Mavor, R A, Parsons M, Heubeck M, Schmitt S (2005) Seabird numbers and breeding success in Britain and Ireland, 2004. UK Nature Conservation no. 29. JNCC, Peterborough

Mitchell PI, Newton SF, Ratcliffe N, Dunn TE (2004) Seabird populations of Britain and Ireland. T. \& A.D. Poyser, London

Phillips RA, Catry P, Thompson DR, Hamer KC, Furness RW (1997) Inter-colony variation in diet and reproductive performance of great skuas Catharacta skua. Mar Ecol Prog Ser 152:285-293

Raclot T, Groscolas R, Cherel Y (1998) Fatty acid evidence for the importance of myctophid fishes in the diet of king penguins, Aptenodytes patagonicus. Mar Biol 132:523-533

Saito H, Kotani Y (2000) Lipids of four boreal species of calanoid copepods: origin of monoene fats of marine animals at higher trophic levels in the grazing food chain in the subarctic ocean ecosystem. Mar Chem 71:69-82

Sydeman WJ, Hobson KA, Pyle P, McLaren EB (1997) Trophic relationships among seabirds in central California: combined stable isotope and conventional dietary approach. Condor 99:327-336

Thompson DR, Lilliendahl K, Solmundsson J, Furness RW, Waldron S, Phillips RA (1999) Trophic relationships among six species of Icelandic seabirds as determined through stable isotope analysis. Condor 101:898-903

Votier SC, Bearhop S, MacCormick A, Ratcliffe NR, Furness RW (2003) Assessing the diet of great skuas, Catharacta skua, using five different techniques. Polar Biol 26:20-26

Votier SC, Furness RW, Bearhop S, Crane JE and 11 others (2004) Changes in fisheries discard rates and seabird communities. Nature 427:727-730

Wanless S, Harris MP, Redman P, Speakman JR (2005) Low energy values of fish as a probable cause of a major seabird breeding failure in the North Sea. Mar Ecol Prog Ser 294: $1-8$

Submitted: March 7, 2006; Accepted: October 17, 2006 Proofs received from author(s): July 2, 2007 